\title{
Calidad de vida de las pacientes con cáncer de mama: cirugía radical versus cirugía conservadora
}

\author{
Quality of life in patients with breast cancer: \\ radical versus conservative surgery in a highly specialized medical unit
}

\author{
J Jesús Medina Valencia, ${ }^{*}$ Wenceslao Guillermo Ángeles Bueno, ${ }^{*}$ \\ Juan Antonio Delgado Vázquez, ${ }^{*}$ Jorge Antonio Lomelí Muñoz,** \\ Joan Arlene Ojeda Babilonia,** Leonora Montes Velázquez**
}

Palabras clave:

Cáncer de mama, calidad de vida, cirugía radical, cirugía conservadora.

Key words: Breast cancer, quality of life, radical surgery, conservative surgery.

* Departamento de Cirugía Oncológica, Unidad Médica de Alta Especialidad (UMAEHE), Centro Médico Nacional de Occidente (CMNO), Guadalajara, Jalisco, México. ** Servicio Social, Departamento de Cirugía-Ginecología, Hospital de Ginecología, CMNO, Guadalajara, Jalisco, México.

\section{RESUMEN}

Introducción: El cáncer de mama es la principal neoplasia maligna en mujeres y la primera causa de muerte por neoplasia en el género femenino en el ámbito mundial, con cerca de 500 mil decesos cada año. Sin embargo, durante su evolución, el enfoque se ha basado sólo en el tratamiento, olvidando el aspecto psicológico de la enfermedad, motivo por el cual se ha tratado de integrar éste en el estudio de la calidad de vida relacionada con la salud, donde se analiza tanto el enfoque clínico postratamiento como las repercusiones físicas y los cambios relacionados con el comportamiento y estado de ánimo, lo que permite destacar el estado funcional de la paciente que refleje su salud física, mental y social. Material y métodos: Estudio trasversal descriptivo de pacientes con cáncer de mama con tratamiento quirúrgico, incluyendo mastectomía o cirugía conservadora, en vigilancia por la consulta externa, atendidas en la UMAE-HE, CMNO durante el periodo comprendido entre el 1 de enero de 2000 y el 31 de diciembre de 2013 en etapas clínicas I a III. Las pacientes fueron evaluadas a partir de la aplicación de un cuestionario del grupo EORTC QLQ-C30 y QLQ-BR23. Resultados: La calidad de vida global de las pacientes fue de 45.5 para mastectomía y 42.7 para cirugía conservadora, con un estado global de salud de 44.1. De todos los parámetros, solo fueron significativos para el apartado de síntomas - específicamente para el estreñimiento- y el impacto económico, los cuales fueron peores para las pacientes con mastectomía. De la misma forma, en el cuestionario específico para cáncer de mama, ninguno de los apartados mostró una diferencia estadísticamente significativa, por lo que se puede asumir una calidad de vida igual entre los diferentes tipos de tratamiento. Conclusión: Las pacientes con cáncer de mama de nuestra institución presentan una menor calidad de vida con respecto a lo descrito en la literatura mundial, lo que requiere de la implementación de programas enfocados en la valoración de estos aspectos durante el abordaje y seguimiento de las pacientes, y no sólo en el tratamiento oncológico.

\section{ABSTRACT}

Introduction: Cancer is the leading malignancy in women, and breast cancer is the leading cause of death from malignancy in females worldwide, with around 500 thousand deaths each year. However, during its evolution the focus has only been on the treatment, forgetting the psychological aspects of the disease. For this reason, this study includes the health-related quality of life, which analyses the postreatment clinical approach, the physical repercussions and the changes associated with behavior and mood, which will enable to highlight a functional status of the patients that reflects their physical, mental and social health. Methods: A descriptive, cross-sectional study conducted on breast cancer patients in clinical stages I to III managed with surgical treatment, including mastectomy or conservative surgery, on follow-up in the Outpatient Clinic of the UMAE-HE, CMNO, during the period between January $1^{\text {st }}, 2000$ and December $31^{\text {st }}$, 2013. The evaluations were based on the questionnaires of the EORTC group QLQ-C30 and QLQ-BR23. Results: The overall quality of life score of the patients was 45.5 for mastectomy and 42.7 for conservative surgery, with an overall health status of 44.1. Of all the parameters, there were only differences in the symptoms section, specifically for constipation, and financial impact, which were worse for patients with mastectomy. Likewise, in the questionnaire specific for breast cancer, no statistically significant differences were observed in any of the sections, from which it can be assumed that the quality of life is similar between the two types of treatment. Conclusion: Patients with breast cancer in this institution have a lower quality of life than that reported in the world literature. This suggests the need to implement programs that focus on these aspects, and not only on the oncological management, during the treatment and follow-up of patients. 


\section{INTRODUCCIÓN}

- $\mathrm{n}$ la actualidad, el cáncer de mama ha - presentado un aumento en su frecuencia hasta convertirse, desde el 2006, en la principal neoplasia de la mujer, tanto en el ámbito nacional como internacional, con un riesgo global de $12.08 \%$ durante toda la vida (una de cada ocho mujeres), con una recurrencia de 13,648 y una mortalidad en México de 5,133 defunciones en 2010, con una tasa de 10.1 defunciones por cada 100,000. ${ }^{1}$ De igual forma, existe un auge en su enfoque, en el que se consideran las implicaciones que tiene sobre la vida de la paciente o, más concretamente, en su calidad de vida.

A partir de 1994, después de la implementación de nuevos programas de detección, se observó un incremento en la frecuencia, la cual se ha mantenido de manera constante, tal y como se muestra en la figura 1. En 1958, la Union for International Cancer Control (UICC) presentó una clasificación clínica del cáncer de mama basándose en el sistema TNM, y en 1977, el American Joint Committee on Cancer (AJCC) publicó la estadificación para el cáncer de mama. Desde entonces, se han realizado revisiones y actualizaciones en función de los avances diagnósticos y terapéuticos que han ido apareciendo hasta la séptima edición de la AJCC (Cuadro I). ${ }^{2}$

Los momentos que pueden ser considerados como críticos en el trascurso de la enfermedad son el diagnóstico, la intervención quirúrgica y el inicio de la quimioterapia y la radioterapia. Sin embargo, existe otro momento estresante para las pacientes, y es el final del tratamiento, cuando tienen que volver a la vida normal. ${ }^{3}$

Por lo general, la evaluación del estado de salud de los individuos se ha basado en la observación o intervención médica, tomando en cuenta los instrumentos tradicionales, como el examen clínico, los laboratorios y estudios de gabinete, entre otros, los cuales se encuentran clasificados como fiables y cuantificables, y se daba poco interés al resto de los instrumentos, que requerían de la percepción subjetiva de los individuos (como en el caso de los cuestionarios de capacidad funcional o síntomas declarados, que se consideraban menos fiables y no cuantificables). ${ }^{4}$ No obstante, algunos de sus parámetros son requeridos para la evaluación integral del paciente. Dentro de los principales perfiles de salud se encuentran el Medical Outcomes Study Short Form 36 (MOS-SF36), probablemente el instrumento más utilizado en la actualidad y del que existen algunas

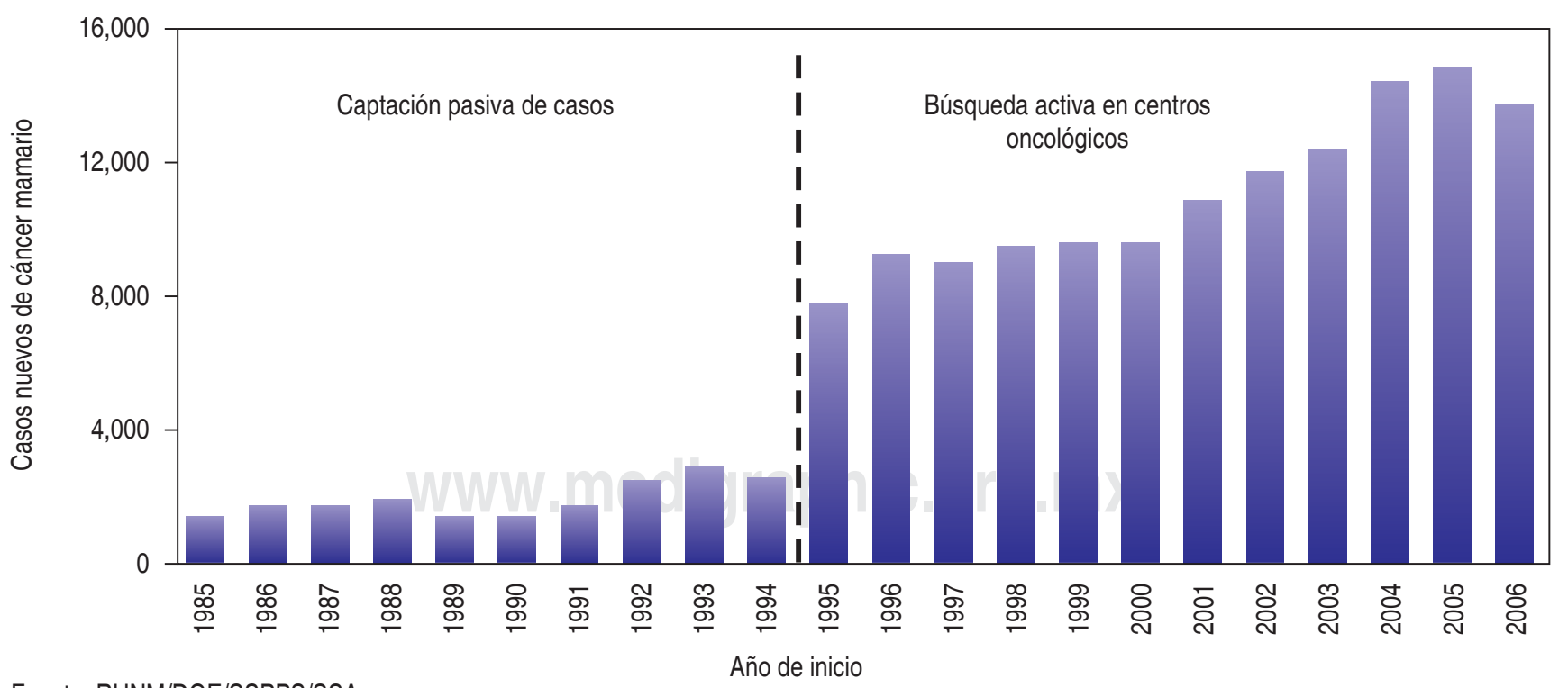

Fuente: RHNM/DGE/SSPPS/SSA.

Figura 1. Casos nuevos de cáncer de mama por año de diagnóstico identificados por el RHNM. México, 1985-2006. 
Cuadro I. Estadificación del cáncer de mama.

\section{Tumor primario}

\begin{tabular}{|c|c|}
\hline TX & No se puede evaluar el tumor primario \\
\hline $\mathrm{T} 0$ & No existe prueba de tumor primario \\
\hline Tis & Carcinoma in situ \\
\hline Tis (CDIS) & Carcinoma ductal in situ \\
\hline Tis (CLIS) & Carcinoma lobulillar in situ \\
\hline Tis (Paget) & $\begin{array}{l}\text { Enfermedad de Paget del pezón que no está relacionada con el carcinoma invasor o carcinoma in situ (CDIS o CLIS) } \\
\text { en el parénquima mamario subyacente. Los carcinomas del parénquima mamario relacionados con la enfermedad de } \\
\text { Paget se clasifican sobre la base del tamaño y las características de la enfermedad parenquimatosa, aunque la presen- } \\
\text { cia de la enfermedad de Paget aún se debería señalar }\end{array}$ \\
\hline $\mathrm{T} 1$ & El tumor mide $\leq 20 \mathrm{~mm}$ en su mayor dimensión \\
\hline T1mi & El tumor mide $\leq 1 \mathrm{~mm}$ en su mayor dimensión \\
\hline Tla & El tumor mide $>1 \mathrm{~mm}$, pero $\leq 5 \mathrm{~mm}$ en su mayor dimensión \\
\hline $\mathrm{T} 1 \mathrm{~b}$ & El tumor mide $>5 \mathrm{~mm}$, pero $\leq 10 \mathrm{~mm}$ en su mayor dimensión \\
\hline T1c & El tumor mide $>10 \mathrm{~mm}$, pero $\leq 20 \mathrm{~mm}$ en su mayor dimensión \\
\hline $\mathrm{T} 2$ & El tumor mide $>20 \mathrm{~mm}$, pero $\leq 50 \mathrm{~mm}$ en su mayor dimensión \\
\hline $\mathrm{T} 3$ & El tumor mide $>50 \mathrm{~mm}$ en su mayor dimensión \\
\hline $\mathrm{T} 4$ & $\begin{array}{l}\text { El tumor mide cualquier tamaño con extensión directa a la pared pectoral o la piel (ulceración o nódulos } \\
\text { cutáneos) }\end{array}$ \\
\hline $\mathrm{T} 4 \mathrm{a}$ & Extensión a la pared torácica que no sólo incluye adherencia o invasión a los músculos pectorales \\
\hline $\mathrm{T} 4 \mathrm{~b}$ & $\begin{array}{l}\text { Ulceración de la piel o nódulos satélites ipsilaterales o edema (incluida la piel de naranja), la cual no satisface el } \\
\text { criterio de carcinoma inflamatorio }\end{array}$ \\
\hline $\mathrm{T} 4 \mathrm{c}$ & Ambos, T4a y T4b \\
\hline T4d & Carcinoma inflamatorio \\
\hline $\mathbf{N}$ & Ganglios linfáticos regionales (clínicos) \\
\hline
\end{tabular}

NX No se pueda evaluar el ganglio linfático regional (por ejemplo, fue extirpado previamente)

N0 Sin ganglios linfáticos regionales palpables

N1 Metástasis palpables a uno o varios ganglios linfáticos homolaterales axilares móviles

N2 Metástasis a ganglio(s) axilares homolaterales fijos entre sí o a otras estructuras, o detectados clínicamente en la cadena mamaria interna homolateral en ausencia de metástasis axilares palpables

N2a Metástasis en ganglio(s) axilares fijos entre sí o a otras estructuras

$\mathrm{N} 2 \mathrm{~b} \quad$ Metástasis clínicamente aparentes en la cadena mamaria interna sin evidencia clínica de metástasis axilares

N3 Metástasis a ganglio(s) linfáticos infraclaviculares homolaterales o a ganglios clínicamente aparentes de la cadena mamaria interna homolateral y en presencia de ganglio(s) axilares palpables o metástasis a ganglio(s) de la región supraclavicular homolateral con o sin ganglios

N3a Metástasis a ganglio(s) infraclavicular homolateral y ganglio(s) axilares

N3b Metástasis a ganglio(s) de la mamaria interna y axilares homolaterales

N3c Metástasis a ganglio(s) supraclaviculares homolaterales

\section{Metástasis a distancia}

$\begin{array}{ll}\text { MX } & \text { No evaluable } \\ \text { M0 } & \text { Sin metástasis a distancia } \\ \text { M1 } & \text { Con metástasis a distancia }\end{array}$


Continúa Cuadro I. Estadificación del cáncer de mama.

\begin{tabular}{cccc}
\hline Estadio & T & $\mathbf{N}$ & M \\
\hline 0 & Tis & N0 & M0 \\
IA & T1 (incluye T1 mi) & N0 & M0 \\
IB & T0 & N1mi & M0 \\
& T1 (incluye T1 mi) & N1mi & M0 \\
IIA & T0 & N1 & M0 \\
& T1 (incluye T1 mi) & N1 & M0 \\
TIB & T2 & N0 & M0 \\
& T3 & N1 & M0 \\
IIIA & T0 & N0 & M0 \\
& T1 (incluye T1 mi) & N2 & M0 \\
& T2 & N2 & M0 \\
& T3 & N1 & M0 \\
& T3 & N2 & M0 \\
IIIB & T4 & N0 & M0 \\
& T4 & N1 & M0 \\
IIIC & T4 & N2 & M0 \\
IV & Cualquier T & N3 & M0 \\
& Cualquier T & Cualquier N & M1
\end{tabular}

versiones reducidas, el perfil de salud de Nottingham (Nottingham Health Profile, NHP) y el perfil de impacto de la enfermedad (Sickness Impact Profile, SIP). ${ }^{5}$ Todos ellos disponen de numerosos estudios de validación en diferentes poblaciones, incluyendo pacientes quirúrgicos de diferentes tipos; se trata de instrumentos registrados cuya utilización requiere la autorización de sus propietarios.

La calidad de vida se ha convertido en una categoría imprescindible para el abordaje integral en el estudio de las enfermedades crónicas. No obstante las variadas posiciones que existen en el análisis de este concepto, todos los autores coinciden en señalar la necesidad de tomar en cuenta las opiniones, vivencias, expectativas y sufrimientos del individuo que enfrenta la realidad de padecer una enfermedad crónica. Así pues, se impone la necesidad de evaluar la enfermedad desde el punto de vista de quien la sufre. ${ }^{6}$

Barbon y Beato encontraron que los sufrimientos o trastornos que aquejan a la mujer con cáncer de mama incluyen frecuentemente ansiedad, insomnio, vergüenza, sentimientos de inutilidad y subvaloración personal. La depresión, reacción emocional muy frecuente en los enfermos crónicos al inicio de su enfermedad, ha sido estudiada por Ford, quien encuentra que los síntomas depresivos se relacionan estrechamente con el estadio de la enfermedad y con el apoyo social. ${ }^{7}$ En Inglaterra, Fenlon estableció un estudio de cohorte de mujeres con diagnóstico reciente de cáncer, tratando de describir la experiencia del dolor musculoesquelético en la entrevista inicial de las participantes y explorar su impacto en la calidad de vida. Refiere la presencia de dolores articulares o musculares, dolores o rigidez en $69 \%$ de las mujeres, lo cual destaca la importancia de una mejor comprensión de estos síntomas y su impacto en la vida de mujeres con cáncer de mama primario, para que los profesionales de la salud estén en mejores condiciones de apoyar a las pacientes y para proporcionar 
información precisa para tomar las decisiones de tratamiento. 8,9

A pesar de que el cáncer de mama ocupa el primer lugar en frecuencia en las mujeres del mundo, se ha visto una disminución en su mortalidad, en parte debido al incremento en los programas de detección temprana y screening (examen), lo que permite llevar a cabo procedimientos quirúrgicos menos agresivos y aumentar la supervivencia de las pacientes; sin embargo, se ha descuidado un punto importante: la evaluación de la calidad de vida de las enfermas. Existen estudios previamente realizados en los que se evalúa la satisfacción de las pacientes, así como la calidad de vida de manera global; sin embargo, ninguno enfocado en la calidad de vida posterior al tratamiento quirúrgico, tomando en cuenta si se realizó una cirugía conservadora o la mujer fue sometida a una mastectomía. La aplicación de escalas de evaluación simples nos permitiría detectar alteraciones importantes en las pacientes y tomar medidas oportunas para reintegrarlas a la sociedad con la mejor situación posible.

Existen estudios sobre la calidad de vida de la paciente con cáncer de mama donde se demuestra que la etapa clínica, el estado civil soltera y el nivel de escolaridad presentan aspectos importantes. Sin embargo, realizan las evaluaciones de manera global, sin tomar en cuenta el tipo de tratamiento quirúrgico, por lo que resulta importante evaluar el impacto psicológico que puede tener una cirugía mutilante en comparación con una cirugía conservadora.

El objetivo de este estudio fue determinar la calidad de vida de las pacientes con cáncer de mama con tratamiento quirúrgico, cirugía radical versus cirugía conservadora, en la Unidad Médica de Alta Especialidad en el Hospital de Especialidades del Centro Médico Nacional de Occidente (UMAE HE CMNO).

\section{MATERIAL Y MÉTODOS}

Se realizó un estudio transversal descriptivo. Se seleccionó a pacientes con cáncer de mama con tratamiento quirúrgico que acudían a control en la consulta externa del Servicio de Ginecología Oncológica en el periodo comprendido entre el 1 de enero de 2000 y el 31 de diciembre de 2013 en la Unidad Médica de Alta Especialidad en el Hospital de Especialidades del Centro Médico Nacional de Occidente (UMAE HE CMNO). Los criterios de inclusión fueron mujeres de entre 16 y 80 años, manejadas en la UMAE, con cáncer de mama en etapas clínicas I a III, con al menos seis meses posteriores al tratamiento quirúrgico (cirugía conservadora o cirugía radical), independientemente de si requirieron hormonoterapia, quimioterapia o radioterapia. Se descartó a pacientes con enfermedad metastásica al momento de su evaluación (etapa clínica IV), cáncer primario en otro sitio y aquéllas en etapa clínica I-III consideradas no candidatas al tratamiento quirúrgico. Todas las pacientes fueron evaluadas con base en la aplicación de los cuestionarios del grupo European Organisation for Research and Treatment of Cancer QLQ-C30 (calidad de vida en pacientes con cáncer en general) y QLQ-BR23 (calidad de vida en pacientes con cáncer de mama). A partir de esta información, se realizaron las mediciones pertinentes de acuerdo con cada variable estadística. Las comparaciones entre los grupos (cirugía conservadora y cirugía radical) se establecieron mediante $\chi^{2}$ en el caso de variables nominales o categóricas; para variables cuantitativas se utilizó T de Student o U de Mann-Whitney según la distribución de la curva. El estudio fue sometido a revisión y aprobación por el Comité Local de Investigación en Salud de la UMAE HE CMNO.

\section{RESULTADOS}

Frecuencia en tratamiento conservador $y$ quirúrgico. Se realizaron un total de 114 encuestas, de las cuales 58 fueron del grupo de mastectomía y 56 del grupo de cirugía conservadora; la edad promedio de la muestra fue de 52.6 años y no hubo diferencia entre los grupos de mastectomía y cirugía conservadora, con 51.8 y 53.3 años, respectivamente.

Ganglio centinela. La realización de ganglio centinela se llevó a cabo en 33 pacientes $(28.9 \%)$, en tres de las cuales se realizó mastectomía y en 30, cirugía conservadora (5.2 y $53.6 \%$ ). Por consiguiente, las 
pacientes con mastectomía tuvieron una mayor frecuencia de linfadenectomías axilares, correspondiendo a $96.6 \%$, en contraparte con el $75 \%$ de las pacientes tratadas con cirugía conservadora.

Quimioterapia, hormonoterapia y radioterapia. No hubo diferencia significativa en cuanto al tratamiento con quimioterapia entre ambos grupos de muestras, que fue del 93.1 y $91.1 \%$ en el tratamiento radical con mastectomía y en la cirugía conservadora,

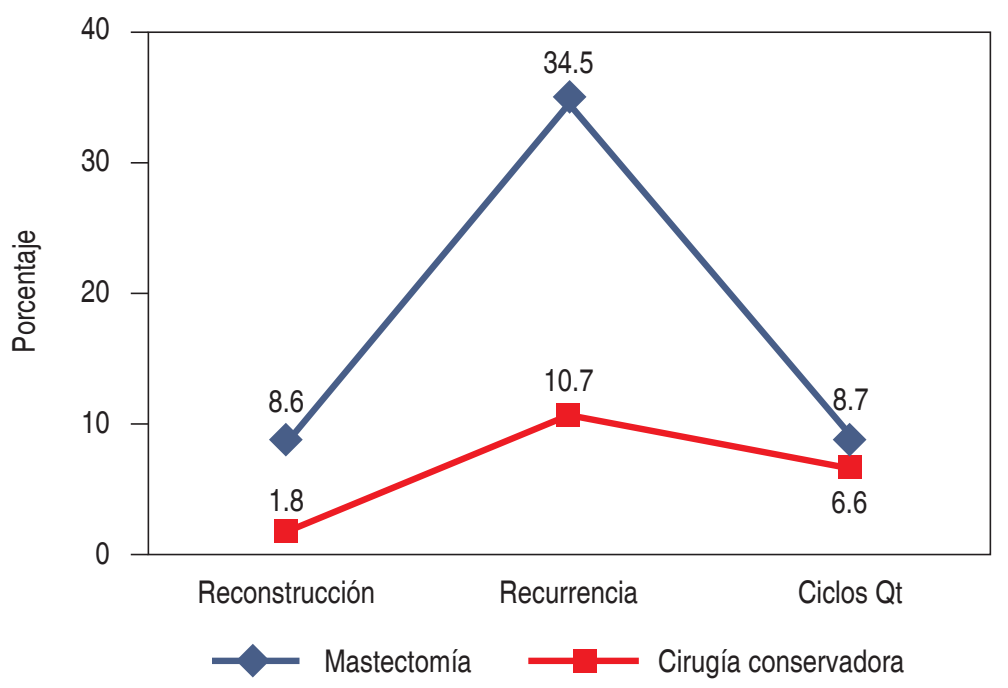

Figura 2. Recurrencia de pacientes con cáncer de mama y ciclos de quimioterapia. respectivamente. En lo que respecta al uso de hormonoterapia, la recibieron 33 pacientes con mastectomía y 26 que tuvieron cirugía conservadora. Hubo una diferencia estadísticamente significativa en cuanto al uso de radioterapia, puesto que una mayor cantidad de pacientes del grupo de cirugía conservadora la recibieron, $94.6 \%$ versus $77.6 \%$, lo cual es comprensible puesto que se considera prácticamente una condición inseparable de la realización de cirugía conservadora. No existió una diferencia en lo que respecta al lado afectado, puesto que un $44.8 \%$ de las mastectomías fueron derechas versus un $57.1 \%$ de las cirugías conservadoras, y de manera global, el lado derecho ocupó un $50.95 \%$ y el izquierdo un $49.05 \%$. De igual forma, no hubo una diferencia en la realización de reconstrucción de las pacientes, independientemente del grupo al que pertenecieron, con un porcentaje de $8.6 \%$ versus $1.8 \%$, sin que se consiguiera una significancia estadística (Figuras 2 y 3).

Recurrencia del cáncer de mama. Un dato importante es la presencia de una mayor recurrencia en el grupo de cirugía radical, con un porcentaje de $34.5 \%$ contra sólo un $10.7 \%$ de las pacientes con cirugía conservadora, lo que se puede explicar por la mayor cantidad de pacientes en etapas

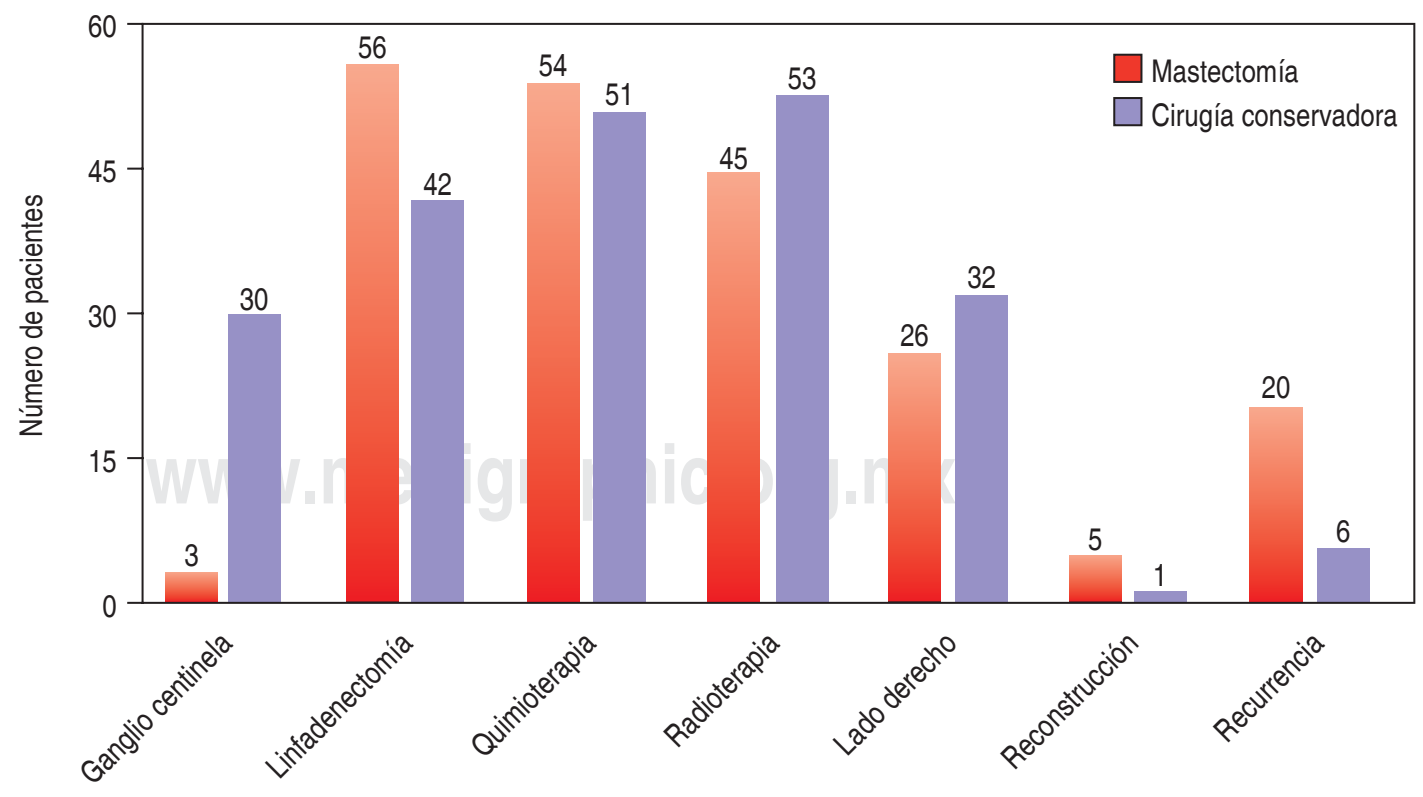


avanzadas de la enfermedad en el grupo de mastectomías.

Estadificación y tratamiento. Existió una diferencia en lo que respecta a las etapas clínicas de las pacientes, ya que aquéllas con cirugía conservadora presentaron etapas clínicas más iniciales, en comparación con las pacientes con mastectomías, las cuales tuvieron etapas clínicas más avanzadas. En general, las pacientes tuvieron una etapa IA en $6.14 \%$, IB $11.95 \%$, IIA $25.4 \%$, IIB en $24.56 \%$, IIIA en $18.42 \%$, IIIB $8.77 \%$ y IIIC en $4.39 \%$, por lo que la mayor cantidad de pacientes se encontraban en EC II, con un total de 50\% versus $\mathrm{ECI}$, de $18 \%$ y EC III, de $31.58 \%$, como se muestra en la figura 4.

Calidad de vida. La calidad de vida global de las pacientes fue de 45.5 para las de mastectomía y 42.7 para las de cirugía conservadora, sin diferencias estadísticamente significativas entre los grupos, con un estado global de salud de 44.1. En general, no hubo una diferencia entre los tipos de tratamiento, puesto que de manera global no presentaron percepciones diferentes en los cuestionarios de calidad de vida. Sólo en el QLQ-30 fueron diferentes para el apartado de síntomas, específicamente para el estreñimiento y el impacto económico, los cuales fueron peores para las pacientes con mastectomía. De la misma forma, en el cuestionario específico para cáncer de mama, ninguno de los apartados mostró una diferencia estadísticamente significativa, por lo que se puede asumir una calidad de vida igual entre los diferentes tipos de tratamiento. Cuando se hizo la evaluación de la calidad de vida de las pacientes con respecto a la realización de ganglio centinela, se vio un estado global de salud similar, con un resultado de 42.43 versus 44.86 , para la realización o no de ganglio centinela, respectivamente. De forma similar a lo observado en la evaluación previa, sólo el apartado de síntomas fue el que logró una diferencia en la evaluación de la diarrea, pero no así en el BR-23, donde se mantuvieron con resultados similares entre ambos grupos.

Calidad de vida postlinfadenectomía. La evaluación de la calidad de vida en las pacientes con o sin linfadenectomía mostró resultados diferentes: fue mejor en aquéllas con cirugía conservadora. De igual forma, los síntomas fueron menores en las pacientes con cirugía conservadora, predominantemente a nivel de la fatiga, así como el dolor, el estreñimiento y el impacto económico.

Quimioterapia, radioterapia, hormonoterapia y calidad de vida. Para el apartado del uso de quimioterapia se logró una diferencia en la calidad de vida, la cual se vio mejor en las pacientes que estaban recibiendo quimioterapia, a pesar de lo que
Figura 4.

Manejo de pacientes según estadificación del cáncer de mama.

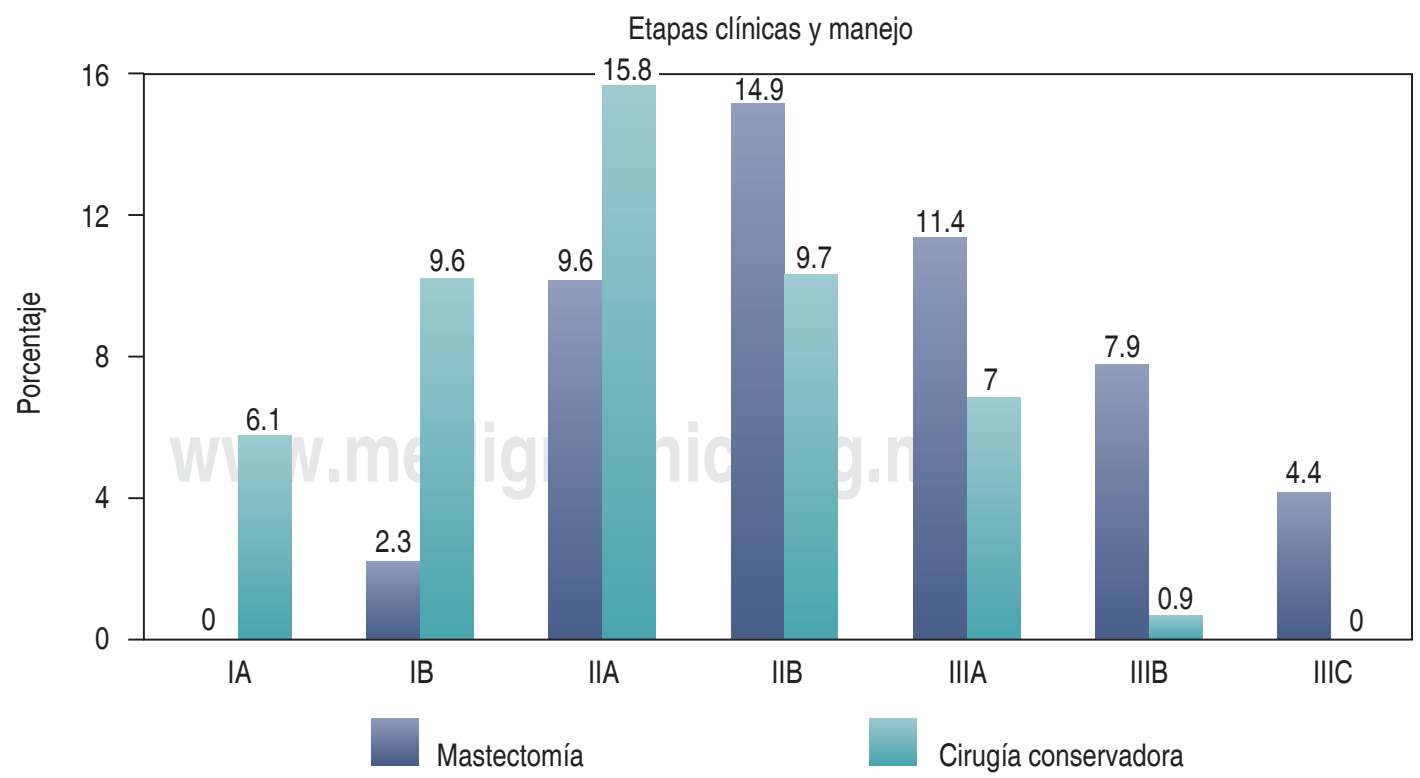


se pudiera pensar, con un estado global de salud de 44.84 contra un 36.11 a favor de las pacientes con quimioterapia. No obstante, el resto de los parámetros permanecieron similares entre ambos grupos en el resto de las escalas. Para la evaluación de la radioterapia, mostraron una calidad de vida similar, de 44.13 versus 44.27 , sin que se apreciara una diferencia en el QLQ-C30. En cambio, cuando se realizó el análisis de la escala específica para el cáncer de mama, se vio una diferencia en el funcionamiento sexual, que fue peor para las pacientes que fueron tratadas mediante radioterapia, aunque sin impacto en el disfrute sexual, sólo en el funcionamiento. El resto de las evaluaciones se mantuvieron con resultados similares. En general, para las pacientes con hormonoterapia no existió diferencia en la calidad de vida ni tampoco en el resto de las variables estudiadas para el QLQ-C30. No obstante, cuando se realizó el análisis de BR-23 hubo una diferencia en los aspectos de la sintomatología, la cual fue específicamente peor en lo que respecta a síntomas en el brazo para las pacientes que recibieron hormonoterapia, pero tenían menor preocupación por la caída de pelo que las pacientes que no recibieron hormonoterapia.

Recurrencia y calidad de vida. Uno de los aspectos con más relevancia es el concerniente a la evaluación de la recurrencia, donde se vieron afectadas una mayor cantidad de variables; tal es el caso de la función física, la cual fue mejor en las pacientes sin datos de recurrencia. De la misma forma en las actividades cotidianas, las cuales fueron peores para las pacientes con recurrencia. Con lo que respecta a la sintomatología, se encontraron variaciones en fatiga, dolor, náuseas y vómito, e impacto económico. Por lo que es uno de los aspectos en los que realmente se vio una marcada diferencia en la calidad de vida de las pacientes.

Importancia de la cirugía reconstructiva. Para las pacientes que tuvieron una cirugía reconstructiva, se vio reflejado el impacto en la calidad de la función sexual, tanto en el disfrute como en el funcionamiento sexual, el cual fue mejor en las pacientes con reconstrucción.

\section{DISCUSIÓN}

En nuestro estudio se demostró que la calidad de vida de las pacientes fue menor a la esperada. La puntuación mediana de la serie obtenida en la escala del QLQ-C30, que mide la percepción del estado global de salud, fue de 44.1, siendo 100 la puntuación correspondiente al estado de máxima salud y calidad de vida relacionada con la salud. Esta puntuación se encuentra por debajo de la media establecida en los artículos internacionales, donde los valores medios en la escala de salud global oscilan entre 58 y $77 . .^{10-13}$ Una posible explicación a estos resultados sería el hecho de que la mayoría de las pacientes en este estudio tenían poco tiempo desde la intervención quirúrgica en el momento de la realización de la encuesta, en algunos casos menos de un año, por lo que aún se encontraban en fases de tratamiento, razón por lo cual podría encontrarse mermada su calidad de vida.

Durante la evaluación se tomaron en cuenta diversas variables. Tal es el caso de la realización de ganglio centinela, la cual se llevó a cabo en $28.9 \%$, tres de mastectomía y 30 de conservadora (5.2 versus 53.6\%). Esto se encuentra relacionado con la posibilidad de realizar procedimientos menos agresivos para las pacientes manejadas en las modalidades de cirugía conservadora. Así mismo, las pacientes con mastectomía tuvieron mayor frecuencia de linfadenectomías, con un total de $96.6 \%$, en contraparte con el $75 \%$ de conservadora.

No hubo diferencia en cuanto a la administración de quimioterapia entre ambos grupos: en el grupo de mastectomía se requirió en un $93.1 \%$ de los casos, mientras que en el grupo de cirugía conservadora se requirió en un $91.1 \%$ de ellos, por lo que probablemente no fue un parámetro que afectó los resultados. En lo que respecta al uso de hormonoterapia, se dio en 33 y 26 pacientes, respectivamente.

Un punto importante a destacar fue lo concerniente al uso de radioterapia, la cual, como era de esperarse, se encontraba en una mayor cantidad en el grupo de cirugía conservadora, con $94.6 \%$ versus $77.6 \%$, debido a 
que las pacientes que son tratadas mediante un procedimiento conservador requieren de la aplicación de radioterapia prácticamente como una condición sine qua non, lo cual, como veremos más adelante, tiene implicación en ciertos aspectos de la evaluación de la calidad de vida.

En relación con la modalidad quirúrgica, no hay muchos estudios sobre la asociación entre el tipo de cirugía y la calidad de vida que incluyan pacientes con un seguimiento mayor de cinco años posteriores a su intervención quirúrgica. Según Dorval, el tipo de cirugía (conservadora versus radical) no tiene un efecto importante a largo plazo en la calidad de vida relacionada con la salud. No obstante, las pacientes menores de 50 años tratadas mediante un procedimiento conservador mostraron menos angustia psicológica que las de ese rango de edad sometidas a mastectomía. En las pacientes mayores de 50 años, ocurrió de forma opuesta. Esto se ve reflejado en los resultados de nuestra evaluación, donde la calidad de vida global de las pacientes fue de 45.5 puntos para las sometidas a mastectomía versus 42.7 para las de cirugía conservadora, sin diferencia estadísticamente significativa, lo que concuerda con lo reportado en otras bibliografías. ${ }^{14,15}$

\section{CONCLUSIÓN}

Las pacientes con cáncer de mama de nuestra institución presentan una menor calidad de vida con respecto a lo descrito en la literatura mundial, lo que requiere de la implementación de programas enfocados en la valoración de estos aspectos, y no sólo en el tratamiento oncológico, durante el abordaje y seguimiento de las pacientes. Sin embargo, no existe diferencia en la calidad de vida relacionada con la salud para las enfermas con cáncer de mama, independientemente del tipo de tratamiento al que sean sometidas.

Se requiere seguimiento y estudios posteriores para las pacientes, puesto que la literatura mundial refiere que su calidad de vida se eleva un año después de terminar el tratamiento, y dado que existen muchas mujeres con seguimiento y tratamiento menor a un año, los resultados podrían estar infravalorados y podríamos tener una mejor calidad de vida en las pacientes.

\section{REFERENCIAS}

1. Robles-Castillo J, Ruvalcaba-Limón E, Maffuz A, Rodríguez-Cuevas S. Cáncer de mama en mujeres mexicanas menores de 40 años. Ginecol Obstet Mex. 2011; 79: 482-488.

2. Singletary SE, Connolly JL. Breast cancer staging: working with the seven edition of the AJCC Cancer Staging Manual. CA Cancer J Clin. 2006; 56: 37-47; quiz 50-51.

3. Porzsolt F, Wolpl CP, Rist CE, Kosa R, Büchele G, Gaus W. Comparison of three instruments (QLQ-C30, SF-36, QWB-7) measuring health-related quality of life/quality of well-being. Psychooncology. 1996; 5: 103-117.

4. Badía X, Salamero M, Alonso J. La medida de la salud. Guía de escalas de medición en español. 3a edición. Barcelona: Edimac; 2002: 125-129.

5. Peiró S. Calidad de vida. In: Ruiz P, Alcalde J, Landa JI, editores. Gestión clínica en cirugía. Madrid: Arán Ediciones; 2005. pp. 321-323.

6. Rabin EG, Heldt E, Hirakata VN, Fleck MP. Quality of life predictors in breast cancer women. Eur J Oncol Nurs. 2008; 12: 53-57.

7. Luxardo N. El cuerpo medido. Escalas y mediciones de la calidad de vida de personas con cáncer. Buenos Aires: Universidad de Buenos Aires (UBA); 2007.

8. Fenlon D, Powers C, Simmonds P, Clough J, Addington-Hall J. The JACS prospective cohort study of newly diagnosed women with breast cancer investigating joint and muscle pain, aches, and stiffness: pain and quality of life after primary surgery and before adjuvant treatment. BMC Cancer. 2014; 14: 467.

9. Sistema Único de Información para la Vigilancia Epidemiológica. México: Dirección General de Epidemiología. Secretaría de Salud; 2011.

10. Janni W, Rjosk D, Dimpfl TH, Haertl K, Strobl B, Hepp F, et al. Quality of life influenced by primary surgical treatment for stage I-III breast cancer — long-term follow-up of a matched-pair analysis. Ann Surg Oncol. 2001; 8: 542-548.

11. Schou I, Ekeberg O, Sandvik L, Hjermstad MJ, Ruland CM. Multiple predictors of health-related quality of life in early stage breast cancer. Data from a year follow-up study compared with the general population. Qual Life Res. 2005; 14: 1813-1823.

12. Härtl K, Janni W, Kästner R, Sommer H, Strobl B, Rack B, et al. Impact of medical and demographic factors on long-term quality of life and body image of breast cancer patients. Ann Oncol. 2003; 14: 1064-1071.

13. Kootstra J, Hoekstra-Weebers JE, Rietman H, de Vries J, Baas P, Geertzen JH, et al. Quality of life after sentinel lymph node biopsy or axillary lymph node dissection in stage I/II breast cancer patients: a prospective longitudinal study. Ann Surg Oncol. 2008; 15: 2533-2541. 
14. Pandey M, Thomas BC, Ramdas K, Ratheesan K. Early effect of surgery on quality of life in women with operable breast cancer. Jpn J Clin Oncol. 2006; 36: 468-472.

15. Greene FL, Sobin LH. The staging of cancer: a retrospective and prospective appraisal. CA Cancer J Clin. 2008; 58: 180-190.
Correspondencia:

J. Jesús Medina Valencia

Lago de Camecuaro Núm. 81,

Col. Fuentes del Pedregal, 38863

Moroleón, Guanajuato, México.

Tel: 4454585356 Cel.: 3311144103

E-mail: metastaxix@hotmail.com 